\title{
Methodological Report
}

\section{Primary mouse renal tubular epithelial cells have variable injury tolerance to ischemic and chemical mediators of oxidative stress}

\author{
Anne C. Breggia ${ }^{1}$ and Jonathan Himmelfarb2,*

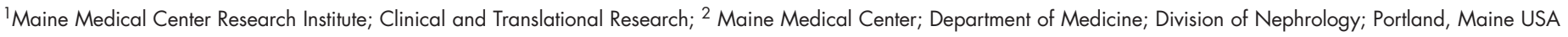

Abbreviations: ATP, adenosine triphosphate; EPO, erythropoietin; HGF, hepatocyte growth factor; IGF-1, insulin growth factor 1; KCM, kidney culture media; LDH, lactate dehydrogenase; LLCPK-1, porcine cortico-tubular epithelial kidney cells; MDCK, Madin-Darby canine kidney; MPT, mitochondrial permeability transition; rhEGF, recombinant human epidermal growth factor; RTEC, renal tubular epithelial cells

Key words: acute kidney injury, ATP, glycolysis, ischemia, lactate dehydrogenase, necrosis, renal tubular epithelial cells

We have developed and evaluated an in vitro culture method for assessing ischemic injury in primary mouse renal tubular epithelial cells (RTEC) in which to explore the pathobiology underlying acute kidney injury. RTEC were predominately of proximal tubule origin which is most susceptible to ischemic injury as compared to other nephron segments. Oxidative stress was induced by chemically depleting ATP using Antimycin A and 2-Deoxy-D-Glucose and by exposing cells to a $1 \%$ oxygen environment. Necrotic injury was assessed by measuring LDH released into culture supernatants. Optimal dose and time of exposure to each injury agent was determined for induction of mild, moderate and severe ischemic injury defined as LDH release of $\leq 20 \%, 21-49 \%$ and $\geq 50 \%$ above baseline respectively. Antimycin A and 2-Deoxy-D-Glucose produced a progressive increase in $\mathrm{LDH}$ release which was time dependent but chemical concentration independent. A $1 \%$ oxygen environment also induced cell injury over time but only if glucose was absent from the culture media. Antimycin A was most effective at inducing oxidative stress causing a mean $\mathrm{LDH}$ release of $61 \%$ at 48 hr compared to $19 \%$ and $50 \% \mathrm{LDH}$ release induced by 2-DeoxyD-Glucose and by exposure to $1 \%$ oxygen respectively at the same 48 hour time point.

The cell culture method described provides several advantages including the use of serum free media and the ability to grow primary cells without matrix support. The LDH assay for injury assessment is reproducible, cost effective, objective and minimizes background cell death. A simple method for the culture and injury of primary mouse renal tubular epithelial cells has thereby been established and provides a useful tool for future investigations of ischemic kidney injury.

\footnotetext{
*Correspondence to: Jonathan Himmelfarb; Maine Medical Center; Nephrology; 22 Bramhall Street; Portland, Maine 04102 USA; Tel.: 207.662.2417; Fax: 207.662.6306; Email: himmej@mmc.org (New contact email: himmej@u.washington.edu)

Submitted: 05/14/08; Revised: 06/19/08; Accepted: 06/23/08

Previously published online as an Oxidative Medicine and Cellular Longevity E-publication: www.landesbioscience.com/journals/oximed/article/6491
}

\section{Introduction}

Despite continued high morbidity and mortality, therapies for the prevention and treatment of acute kidney injury are still lacking. The majority of clinical cases of acute tubular necrosis are caused by ischemic rather than toxic injury. ${ }^{1}$ Renal ischemia-reperfusion injury is characterized by significant depletion of ATP and a reduction in tissue oxygen content that is accompanied by increases in intracellular calcium. ${ }^{2}$ In vitro, primary renal tubular epithelial cells have previously been shown to die of apoptosis or necrosis depending upon the severity of ATP depletion. Necrosis is characterized by rapid metabolic collapse that leads to early loss of plasma membrane integrity and the release of cytosolic contents including proteins such as lactate dehydrogenase (LDH). ${ }^{3} \mathrm{LDH}$ is an enzyme which catalyzes the conversion of lactate to pyruvate at a rate determined by positive feedback inhibition and is retained in the cytosol unless the cell membrane is compromised. While necrotic tissue injury is often evaluated by quantifying the amount of $\mathrm{LDH}$ released into blood plasma, LDH release is not specific for necrotic cell death. Therefore the amount of LDH release attributable to necrotic verses apoptotic cell death cannot be discerned by quantifying LDH release.

Therapies aimed at blocking apoptosis only and which do not target necrosis may be ineffective strategies in the treatment of acute kidney injury. In vitro models of reproducible injury to renal tubular epithelial cells are necessary for understanding renal damage and repair mechanisms and for testing potential therapies.

The purpose of this study was to establish a consistent method for evaluating mild, moderate and severe ischemic injury to primary mouse renal tubular epithelial cells (RTEC).

\section{Results}

Cell culture of renal tubular epithelial cells. Optimal conditions for the culture of primary renal tubule epithelial cells (RTEC) were successfully established as seen by characteristic cobblestone morphology of epithelial cells (Fig. 1C). Proximal tubule epithelial cells are the most susceptible to ischemic injury. The cultured epithelial cells, although a heterogeneous population, were predominantly of proximal tubule origin as seen by fluorescent staining with 
megalin, a proximal tubule brush border specific marker (Fig. 1D). Loss of adherens junctions is an early event in ischemic injury to tubular epithelial cells. As shown in Figure 1E-F, RTEC displayed prominent E-cadherin staining under normoxic conditions which was diminished upon exposure to hypoxia.

Antimycin A depletion of ATP. The time of exposure rather than the concentration of Antimycin A largely determined the extent of injury to RTEC. Antimycin A added to cell cultures at $0.5 \mu \mathrm{M}, 1.0$ $\mu \mathrm{M}, 2.0 \mu \mathrm{M}$ and $3.0 \mu \mathrm{M}$ resulted in $\mathrm{LDH}$ release ranging from $14-25 \%$ at $12 \mathrm{hr}$ to $46-52 \%$ at $48 \mathrm{hr}$. Baseline LDH release in the presence of vehicle media (DMEM without dextrose/1\%ETOH) was determined for each time point and ranged from 16\% at $12 \mathrm{hr}$ to $20 \%$ at $48 \mathrm{hr}$ (Fig. 2A). In independent experiments, addition of $2 \mathrm{uM}$ Antimycin A to cell cultures resulted in $\mathrm{LDH}$ release ranging from $22 \%$ at $12 \mathrm{hr}$ to $61 \%$ at $48 \mathrm{hr}$ following subtraction of baseline LDH release (Fig. 2B).

2-Deoxy-D-Glucose depletion of ATP. There was no significant difference in injury induced by 2-Deoxy-D-Glucose added at 4 $\mathrm{mM}, 5 \mathrm{mM}, 6 \mathrm{mM}$ and $8 \mathrm{mM}$ concentrations at each time point tested. Baseline $\mathrm{LDH}$ release due to vehicle media (DMEM without dextrose) ranged from $13 \%$ at $12 \mathrm{hr}$ to $17 \%$ at $48 \mathrm{hr}$ (Fig. 3A). Exposure of cells to $4 \mathrm{mM} 2$-Deoxy-D-Glucose for $48 \mathrm{hr}$ resulted in a $19 \% \mathrm{LDH}$ which was $11 \%$ greater than the $17 \%$ baseline $\mathrm{LDH}$ release at $48 \mathrm{hr}$ (Figure 3B).

Chamber hypoxia depletion of ATP. As the environmental oxygen concentration in the hypoxic chamber was constant, the amount of injury induced by the chamber was dependent upon the amount of glucose in the culture media and the exposure time to hypoxia. There was little difference in the $\mathrm{LDH}$ release between cultures with media containing no dextrose or $0.5 \mu \mathrm{M}, 1.0 \mu \mathrm{M}$ and $10 \mu \mathrm{M}$ concentrations of dextrose at the $12 \mathrm{hr}$ and $18 \mathrm{hr}$ time points. Increased LDH release occurred with $0.5 \mu \mathrm{M}$ of dextrose and a $24 \mathrm{hr}$ exposure time as well as no dextrose at the $24 \mathrm{hr}$ and $48 \mathrm{hr}$ time points. Vehicle media (DMEM without dextrose) resulted in baseline LDH release of $13 \%$ at $12 \mathrm{hr}$ to $17 \%$ at $48 \mathrm{hr}$ (Fig. $4 \mathrm{~A}$ ). As shown in Figure 4C, a time course using DMEM without dextrose resulted in an $\mathrm{LDH}$ release of $21 \%$ at $24 \mathrm{hr}$ and $50 \%$ at $48 \mathrm{hr}$ following subtraction of baseline LDH release for these time points which was 12\% and 17\% respectively. A summary of the conditions for ischemic injury are shown in Table 1.

\section{Materials and Methods}

Mice. The C57B16 strain was chosen as the source for RTEC culture as they are most often used as the background for genetic manipulation. Mice were purchased from Jackson Laboratories, Bar Harbor, ME. All animal protocols were approved by the Institutional Animal Care and Use Committee of Maine Medical Center Research Institute, Scarborough ME. All mice were male and 8-16 weeks of age at the time of testing.

Culture of primary renal tubular epithelial cells. Mouse primary proximal tubules were recovered, dissociated and digested as previously described. ${ }^{4}$ Mice were anesthetized with Isoflurane and sacrificed by cervical dislocation. Kidneys were immediately removed and placed in cold $\left(0^{\circ} \mathrm{C}\right)$ Hanks Balanced Salt Solution (HBSS) (Invitrogen Corp., Carlsbad, CA) with 1\% antibiotic/antimycotic (Sigma-Aldrich, St. Louis, MO). The renal capsule was removed, and the kidney sagittally cut into two halves. The medulla was

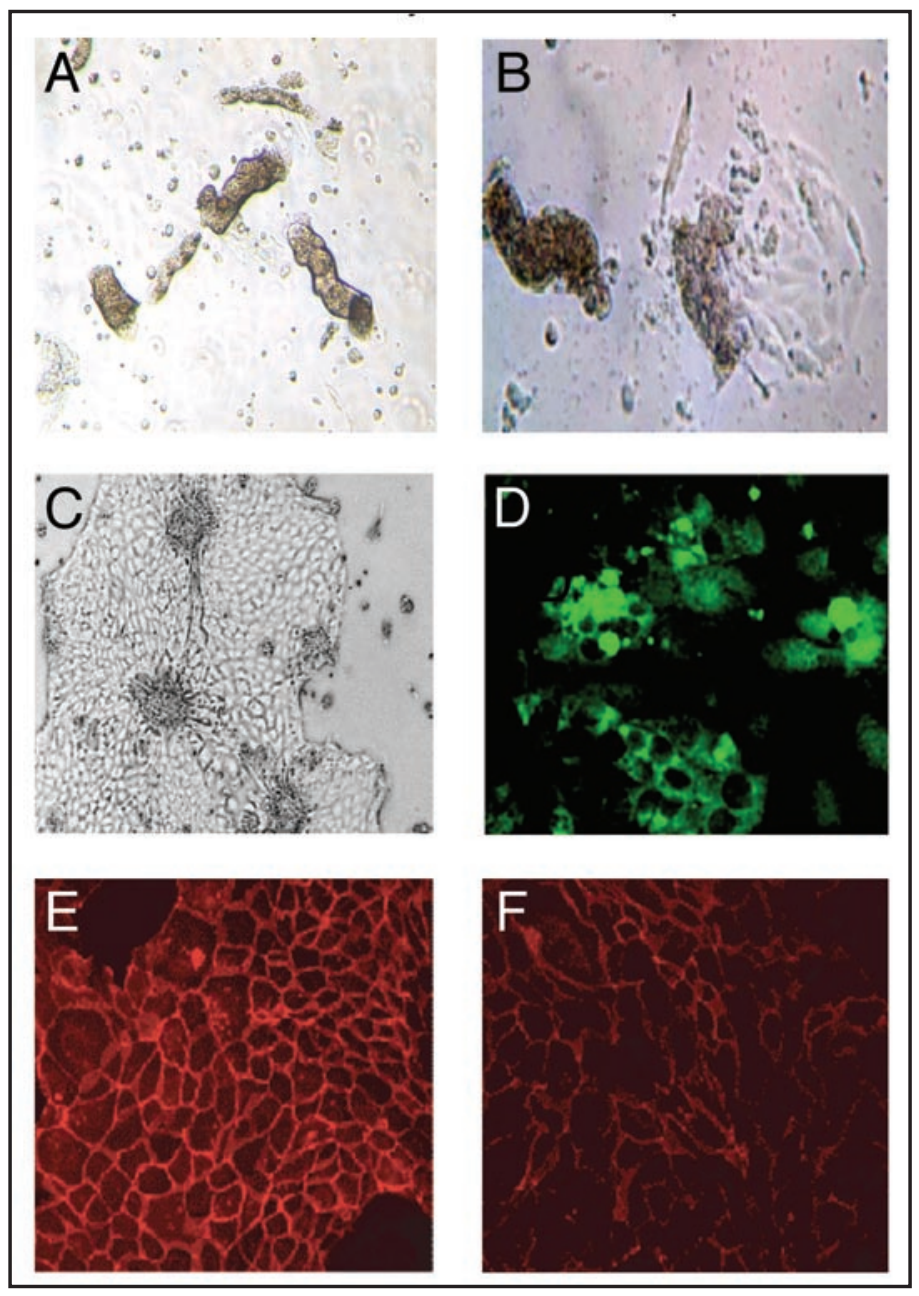

Figure 1. Culture of mouse primary renal tubule epithelial cells. (A) Kidney cortices, abundant in proximal tubular epithelial cells, were digested, resuspended in kidney culture media $(\mathrm{KCM})$ and plated at a previously determined optimal density. (B) On day 2 of culture, tubular epithelial cells migrated out of the tubes and proliferated, forming patches on the plate surface. (C) Individual patches of epithelial cells merged and the cultures reached $90 \%$ confluency on day $7-10$. (D) Epithelial cells were identified as originating from proximal tubules by the brush border specific marker megalin fluorescently tagged with Alexa fluor 488. (E) Under normoxic conditions, E cadherin was prominent on tubular epithelial cells. (F) Upon exposure to hypoxic (1\% oxygen) conditions, E-cadherin staining was diminished.

dissected and discarded from each half. The remaining cortical tissue was minced and transferred to $10 \mathrm{~mL}$ HBSS containing Collagenase (Worthington Biochemical Corp., Lakewood, N.J.) at 200 units/mL and Soybean Trypsin Inhibitor (Invitrogen Corp.) added at the same weight as determined for the Collagenase. Tubules were incubated at $37^{\circ} \mathrm{C}$ while rotating at $70 \mathrm{RPM}$ for 15 minutes. The tubule suspension was mixed with a $10 \mathrm{~mL}$ pipet and returned to the incubator for an additional 15 minutes. Following digestion, tubules from each kidney were mixed again and the tubule suspension divided into two $15 \mathrm{~mL}$ conical tubes with $5 \mathrm{~mL}$ per tube (two tubes of $5 \mathrm{~mL}$ each per kidney). Density sedimentation with horse serum was used to inactivate enzymes and enrich for the proximal tubules. To accomplish this, $5 \mathrm{~mL}$ of sterile, heat inactivated horse serum (Invitrogen Corp.) was added to each tube and the tube vortexed for 30 seconds. Tubules were allowed to sediment for one minute. After 


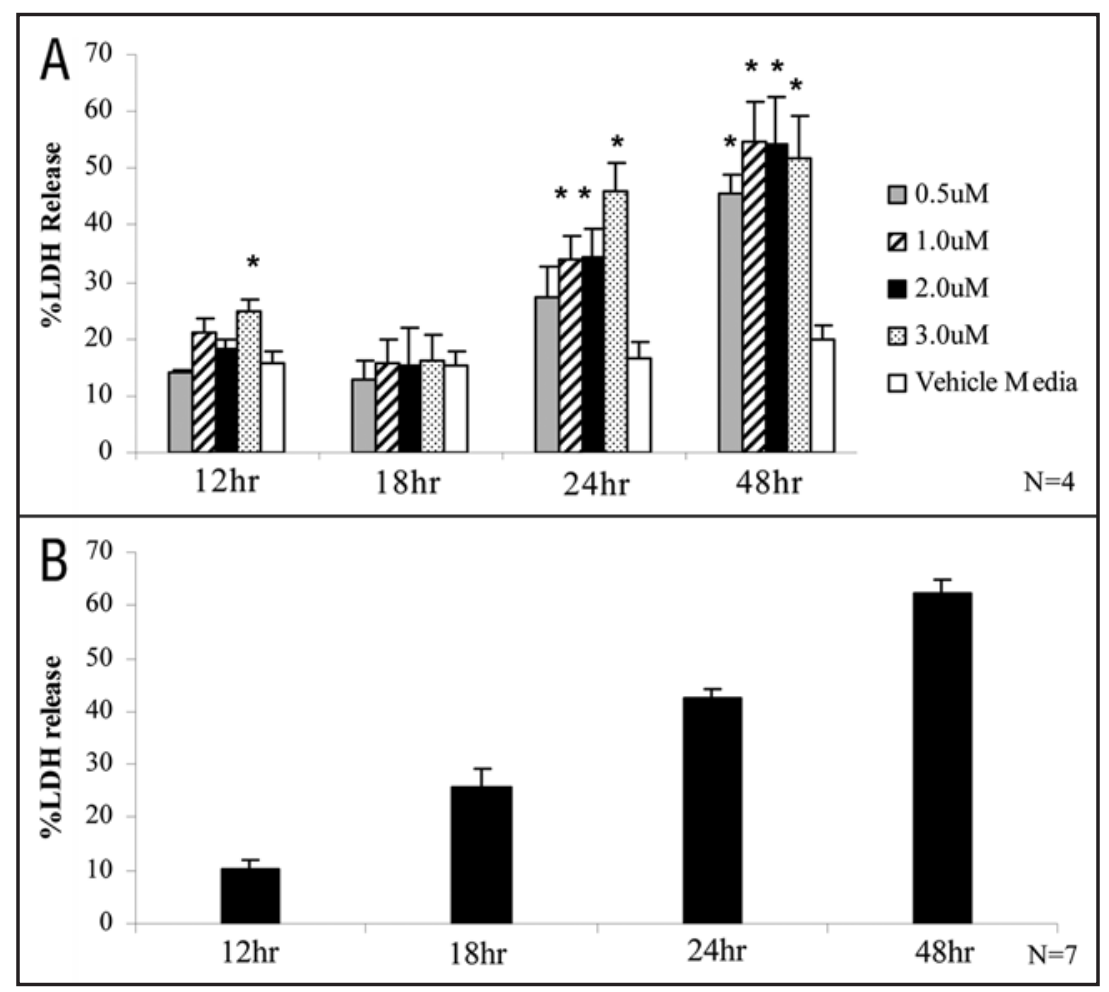

Figure 2. Antimycin A depletion of ATP. Antimycin A was added to cell cultures and injury assessed by the $\mathrm{LDH}$ released into culture supernatants. (A) The $0.5 \mu \mathrm{M}$ concentration did not significantly increase the LDH release unless incubated for $48 \mathrm{hr}(\mathrm{p}<0.05)$. In contrast, the $1.0 \mu \mathrm{M}, 2.0 \mu \mathrm{M}$ and $3.0 \mu \mathrm{M}$ concentrations produced significant injury at $24 \mathrm{hr}$ and $48 \mathrm{hr}(\mathrm{p}<0.01)$. The LDH release in the presence of media control did not significantly increase over time and ranged from $16 \%$ at $12 \mathrm{hr}$ to $20 \%$ at $48 \mathrm{hr}$. (B) A 2 $\mu \mathrm{M}$ concentration of Antimycin A produced LDH release ranging from $10 \%$ at $12 \mathrm{hr}$ to $62 \%$ at $48 \mathrm{hr}$ above baseline.

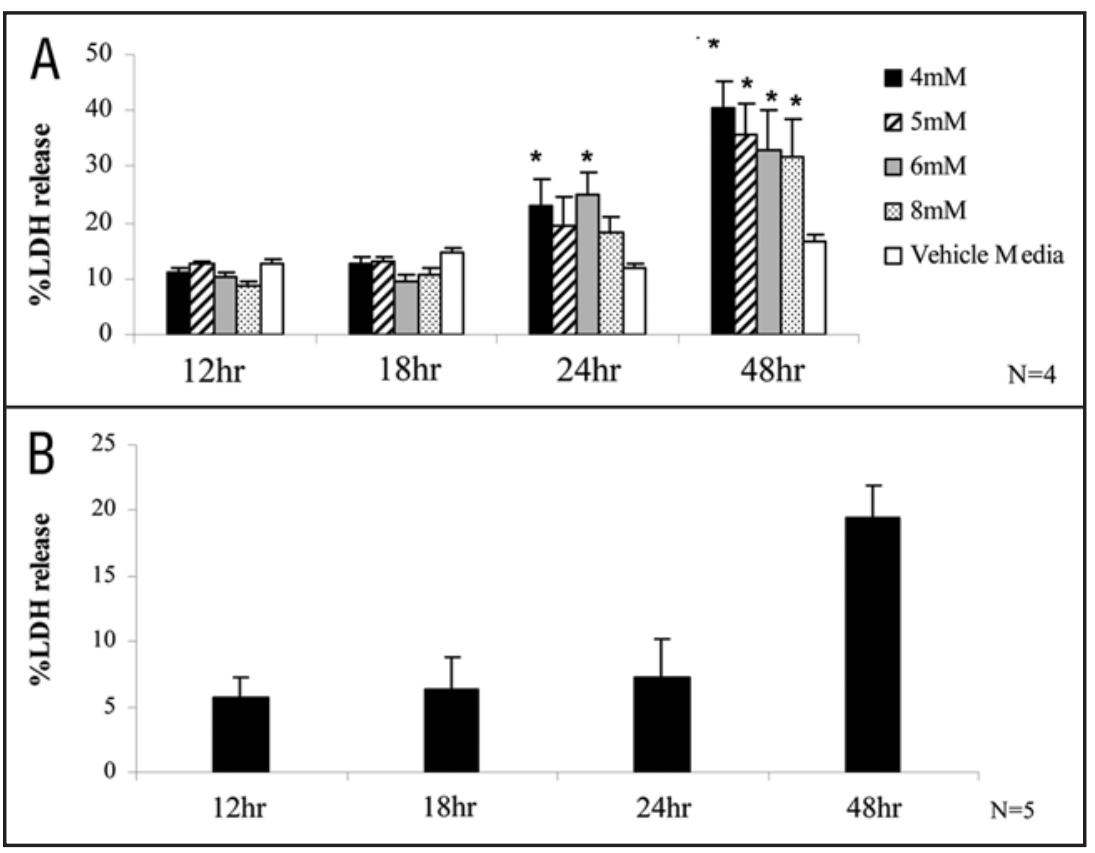

one minute, the supernatant containing the proximal tubules was removed, transferred to another tube and centrifuged for 7 minutes at $200 \mathrm{~g}$. Tubules were washed once with $10 \mathrm{~mL}$ of HBSS and centrifuged at $200 \mathrm{~g}$ for 7 minutes. Tubules were then combined and resuspended in $60 \mathrm{~mL}$ of Kidney Culture Media (KCM) which consisted of DMEM/F-12 culture media (Invitrogen Corp.) containing insulin/transferrin/selenium (Invitrogen, $5 \mu \mathrm{g} / \mathrm{mL}, 2.75 \mu \mathrm{g} / \mathrm{mL}$ and $3.35 \mathrm{ng} / \mathrm{mL}$ respectively), plus $2.0 \mu \mathrm{g} / \mathrm{mL}$ APO transferrin (Sigma-Aldrich), $40 \mathrm{ng} / \mathrm{mL}$ $(0.11 \mu \mathrm{M})$ hydrocortisone (Sigma-Aldrich), $0.01 \mu \mathrm{g} / \mathrm{mL}$ recombinant human epidermal growth factor (rhEGF from R\&D Systems, Minneapolis, MN), and 1\% antibiotic/antimycotic solution (Sigma-Aldrich, 10,000 units/ $\mathrm{mL}$ penicillin, $0.1 \mathrm{mg} / \mathrm{mL}$ streptomycin and $0.25 \mu \mathrm{g} /$ $\mathrm{mL}$ amphotericin B). Dilutional studies were performed previously to determine the volume of resuspended tubules needed for each plating format to ensure optimal growth. Tubules were plated on Nunclon-treated tissue culture plates (Nalgene/Nunc International, Rochester, NY) in 96 well $(200 \mu \mathrm{L} /$ well $), 48$ well $(1.0 \mathrm{~mL} /$ well $)$, 24 well $(1.5 \mathrm{~mL} /$ well $)$ or 6 well $(3 \mathrm{~mL} /$ well $)$ plate formats. Cultures were incubated at $37^{\circ} \mathrm{C}$ with $5 \% \mathrm{CO}_{2}$. Culture media was replaced initially at 24 hours and subsequently every 48-72 hours using KCM without rhEGF.

Ischemic injury induction. Ischemic injury was induced using Antimycin A, 2-Deoxy-D-Glucose or chamber hypoxia on day 7-10 when RTEC cultures had grown to $80-90 \%$ confluency. Cells were incubated in a $200 \mu \mathrm{L}$ volume of either vehicle media (for determination of baseline LDH release) or injury inducing agents at $37^{\circ} \mathrm{C}$ with $5 \% \mathrm{CO}_{2}$ for $12 \mathrm{hr}, 18 \mathrm{hr}, 24 \mathrm{hr}$ and $48 \mathrm{hr}$.

Antimycin A. $0.5 \mu \mathrm{M}, 1.0 \mu \mathrm{M}, 2.0 \mu \mathrm{M}$ and 3.0 $\mu \mathrm{M}$ concentrations of Antimycin A in DMEM without dextrose (Invitrogen) were prepared from a $200 \mu \mathrm{M}$ stock solution in $95 \%$ ETOH. Vehicle control media was DMEM without dextrose and with $1 \% \mathrm{ETOH}$.

2-Deoxy-D-Glucose. $4 \mu \mathrm{M}, 5 \mu \mathrm{M}, 6 \mu \mathrm{M}$ and $8 \mu \mathrm{M}$ concentrations of 2-Deoxy-D-Glucose were prepared in DMEM without dextrose. Vehicle control media was DMEM without dextrose.

Hypoxia. A hypoxic (1\% oxygen) environment was achieved using the Modular Incubator Chamber (Billups-Rothenberg Inc., Del Mar, CA). To do this, each chamber was purged for 4 minutes with a certified gas mixture consisting of $5 \% \mathrm{CO}_{2}$ and $94 \%$ Nitrogen. Chambers were placed in a $37^{\circ} \mathrm{C}$ incubator for 30

Figure 3. 2-Deoxy-D-Glucose depletion of ATP. 2-Deoxy-DGlucose was added to cell cultures and injury assessed by the $\mathrm{LDH}$ released into culture supernatants. (A) At $24 \mathrm{hr}$, the $4 \mu \mathrm{M}$ and $6 u M$ concentrations significantly increased the $\mathrm{LDH}$ release. All concentrations of 2-Deoxy-D-Glucose significantly increased the LDH release at $48 \mathrm{hr}(p<0.02)$. The LDH release in the presence of media control did not significantly increase over time and ranged from $13 \%$ at $12 \mathrm{hr}$ to $17 \%$ at $48 \mathrm{hr}$. (B) A $4 \mathrm{mM}$ concentration of 2-Deoxy-D-Glucose produced an $\mathrm{LDH}$ release ranging from $6 \%$ at $12 \mathrm{hr}$ to $19 \%$ at $48 \mathrm{hr}$ above baseline. 
minutes, removed and re-purged for an additional 4 minutes and then returned to the incubator for the times indicated above. Cell culture media for chamber hypoxia experiments was DMEM without dextrose, or $0.5 \mu \mathrm{M}, 1.0 \mu \mathrm{M}$ and $10 \mu \mathrm{M}$ of dextrose.

Controls. Baseline injury due to incubation of cell cultures in vehicle media (DMEM without dextrose for 2-Deoxy-D-Glucose and chamber hypoxia or DMEM without dextrose and with 1\% ETOH for Antimycin A) was determined by incubation at $37^{\circ} \mathrm{C}$ with $5 \%$ $\mathrm{CO}_{2}$ under ambient air (normoxic) conditions for 12 hr, $18 \mathrm{hr}, 24 \mathrm{hr}$ and $48 \mathrm{hr}$.

LDH assay. Ischemic injury was assessed with the Non-Radioactive Cytotoxicity Assay (Promega, Madison, WI) according to a modification of the manufacturers' protocol. Following treatment, $200 \mu \mathrm{L}$ of supernatants (total volume in each well) were removed from culture test or control wells and transferred to a 96 well plate. Cells were then lysed by adding 20 $\mu \mathrm{L}$ of $1 \%$ Triton-X lysis solution to each well for 10 minutes at room temperature. $180 \mu \mathrm{L}$ of PBS was then added and the lysates transferred to a separate 96 well plate. Each plate was centrifuged at 2000 RPM for 10 minutes. A $50 \mu \mathrm{L}$ volume was removed from each well for reaction with the LDH substrate. Optical density (OD) was read on a MRXTC Revelation spectrophotometer (Dynex Laboratories, Chantilly, VA) at a wavelength of $490 \mathrm{~nm}$. An LDH positive control (Promega) was tested in each assay. Each test was performed in triplicate and the average of triplicate wells used in the final calculations. The LDH released from necrotic cells due to injury was compared to the total $\mathrm{LDH}$ release $\mathrm{LDH}$ in the supernatant plus $\mathrm{LDH}$ in the supernatant of the lysate) and calculated using the formula:

\% LDH Release $=$ Spontaneous LDH Release/Maximum LDH Release (Supernatants + Lysate Supernatants) x 100.

Statistical analysis. All values are expressed as means \pm SEM. The mean LDH release produced by each concentration of injury agents at each time point was analyzed by ANOVA and the unpaired Student's T test for continuous data. Significance was determined by comparing LDH released from RTEC due to injury to the $\mathrm{LDH}$ released due to vehicle media at each time point tested. A $p$ value of $\leq 0.05$ was considered significant.

\section{Discussion}

In order to study mechanisms of injury and repair during ischemic injury in the kidney we have utilized a simple method for the culture of mouse primary renal tubular epithelial cells (RTEC) and for induction of injury that results in necrosis of RTEC.

The renal tubular epithelial cell population, although heterogeneous, was identified as primarily of proximal tubule origin. Proximal tubule epithelial cells are polarized in the basal-lateral and apical surface membranes. Tight junctions consisting of $\mathrm{ZO}-1, \mathrm{ZO}-2$, occludin and cingulin are crucial in maintaining cell polarity. ${ }^{5}$ Adherens junctions, consisting predominantly of
E-cadherin, maintain cell-cell contacts and are linked to the actin cytoskeleton by $\beta$-catenin. ${ }^{6}$ Ischemic injury results in loss of epithelial cell polarity, disruption of the actin cytoskeleton and disassembly of junctional complexes. Detachment of both viable and non viable cells from the lumen causes renal tubular obstruction thus exacerbating ischemic injury. ${ }^{5}$ The assembly and maintenance of both tight junctions and adherens junctions is controlled by Rho-GTPase proteins. ${ }^{7}$ Ischemic injury resulting in ATP depletion has been shown to inhibit Rho-GTPase signaling while activation of Rho protects tight junctions. ${ }^{8}$ Rho-GTPases are members of the Ras family of $\mathrm{p} 21 \mathrm{G}$ proteins and act as molecular switches for a variety of downstream biological processes by cycling between an

\section{Table 1 Summary of injury conditions}

\begin{tabular}{lcccc}
\hline Injury Agent & Time & N & Mean LDH Release & SEM \\
$2 \mu$ M Antimycin A & $12 \mathrm{hr}$ & 7 & $10 \%$ & 4.72 \\
& $18 \mathrm{hr}$ & 7 & $24 \%$ & 3.25 \\
& $24 \mathrm{hr}$ & 7 & $45 \%$ & 3.40 \\
& $48 \mathrm{hr}$ & 7 & $61 \%$ & 2.51 \\
Chamber Hypoxia & $24 \mathrm{hr}$ & 4 & $21 \%$ & 2.53 \\
& $48 \mathrm{hr}$ & 4 & $50 \%$ & 3.01 \\
2-Deoxy-D-Glucose & $48 \mathrm{hr}$ & 5 & $19 \%$ & 2.55
\end{tabular}

$2 \mu \mathrm{M}$ Antimycin A produced a time dependent increase in the LDH release, inducing injury which ranged from $22 \%$ to $61 \%$. Using media without dextrose, the LDH release in cells subjected to chamber hypoxia was $21 \%$ at $24 \mathrm{hr}$ and $50 \%$ at $48 \mathrm{hr}$. $4 \mathrm{mM}$ 2-Deoxy-D-Glucose produced a 19\% LDH release at $48 \mathrm{hr}$. $\mathrm{N} \#=\#$ of experiments $=\#$ of mice. 
active state bound to GTP and an inactive state bound to GDP.? Tubular epithelial cells die by apoptosis or necrosis depending on the severity and duration of ischemic injury. ${ }^{10}$ While ischemia causes depletion of both ATP and GTP, GTP depletion has been shown to result in apoptosis whereas ATP depletion results in necrosis. ${ }^{11}$ The mechanism responsible for this difference has not been determined. Apoptosis was not assessed in this study, however, it could be theorized that, based on the long exposure necessary ( $48 \mathrm{hr}$ ) for induction of mild injury (19\% LDH release) by 2-Deoxy-D-Glucose, apoptosis (GTP depletion) rather necrosis (ATP depletion) accounted for the majority of injury incurred. Further studies measuring cellular GTP and ATP following injury induction by each agent would be needed to clarify this theory. Antimycin A has been previously shown to markedly decrease ATP stores. ${ }^{12}$

In vivo, the proximal tubules, and in particular the $\mathrm{S} 3$ segment, are the most susceptible to ischemic injury as they are highly metabolic but have a low glycolytic capacity due to a low activity of glycolytic enzymes. ${ }^{13}$ In freshly isolated proximal tubules, this is evident by the rapid decline in ATP concentrations in spite of glucose availability and by the lack of lactate production either in the presence or absence of inhibitors of mitochondrial respiration. ${ }^{14}$ Proliferating cultures of proximal tubules have been shown to revert from gluconeogenic to glycolytic metabolism. ${ }^{15}$ The underlying mechanisms responsible for this phenomenon have not been determined but appear to be unrelated to cellular hypoxia, a decrease in ATP, or a high glucose environment. ${ }^{16}$ Glycolytic activity may explain why, in spite of the absence of glucose in vehicle control media, the baseline LDH release was low $(\leq 20 \%)$ and not significantly increased over time. In this study, a high concentration of glucose $(10 \mathrm{mM})$ protected cells from hypoxia-induced cell death while lower concentrations (0.5 $\mathrm{mM}$ and $1.0 \mathrm{mM}$ ) did not. Removal of dextrose from the culture media resulted in $21 \% \mathrm{LDH}$ release from cells exposed to hypoxic air conditions for 24 hours. Moderate-severe necrotic injury $(\geq 50 \%$ $\mathrm{LDH}$ release) was only achieved by a $48 \mathrm{hr}$ exposure to hypoxia. Glycolysis has been shown to be upregulated in rabbit proximal tubules by minimal glucose in culture media $(0.5 \mathrm{mM}$ or $5.0 \mathrm{mM})$ and decreased by high concentrations $(25 \mathrm{mM}) .{ }^{16}$ Hydrocortisone and insulin have also been shown to increase glycolytic activity. ${ }^{17,18}$ Both of these hormones were absent from the vehicle media used for injury induction and therefore not considered as factors for increases in glycolytic activity.

2-Deoxy-D-Glucose is a glucose analog in which the 2-hydroxyl group is replaced by hydrogen. 2-Deoxy-D-Glucose reduces ATP by acting as a competitive inhibitor of glycolysis and by undergoing extensive phosphorylation thereby reducing ATP stores. ATP is expended in the metabolism of 2-Deoxy-D-Glucose but, in contrast to glucose metabolism, is not replenished. ${ }^{19}$ A $48 \mathrm{hr}$ exposure to 2-Deoxy-D-Glucose was needed in order to induce $\mathrm{LDH}$ release of $19 \%$ above baseline. Proximal tubules possess the enzymes necessary for gluconeogenesis and it is therefore likely that amino acids present in the culture media were used to make glucose for ATP production. The small amino acid glycine, a component of the culture media used for injury induction, has been shown to significantly protect against injury to cells in vitro. In isolated rat and rabbit proximal tubules, glycine reduced injury induced by hypoxia, metabolic inhibitors, ouabain, ionomycin and phosphate depletion. ${ }^{20-24}$ The mechanism by which glycine protects cells against ischemic injury is not known but is not related to glycine metabolism, maintenance of cellular ATP, potassium or calcium levels, alterations in intracellular $\mathrm{pH}$, inhibition of phospholipases or removal of free fatty acids. $^{23,25-27}$ Glycine is a required component of tissue culture media as cells cannot be sustained without it.

The mitochondrial permeability transition (MPT), a porous defect of the inner mitochondrial membrane, as well as cytochrome $\mathrm{c}$ release have been identified as potential mechanisms for mitochondrial damage and cell death. ${ }^{28}$ Neither of these has been identified in the proximal tubule, however, and although the mitochondrial lesion can be lethal, cell death is predominantly by the glycine sensitive plasma membrane lesion that causes necrosis and rapid LDH release. ${ }^{13}$ Reoxygenated tubules demonstrate defects in complex I of the mitochondrial respiratory chain while retaining function of complexes II, III and IV. Complex I defects may result in the formation of a precursor lesion that eventually leads to MPT. Complex I defects can be bypassed by metabolism of succinate for electron transport to complex III via complex II (succinate dehydrogenase) provided oxygen is available as the final electron acceptor. Providing substrates such as $\alpha$-ketoglutarate under aerobic conditions improve mitochondrial function by resumption of oxidative phosphorylation which generates ATP to prevent plasma membrane damage and LDH release. ${ }^{28}$ Antimycin A, produced by streptomyces bacteria and used commercially as an antibiotic, prevents ATP production by binding to the cytochrome bc1 complex (Complex III) of the electron transport chain thus interfering with the oxidation of Coenzyme $\mathrm{Q}$ and causing irreversible defects in aerobic respiration in the mitochondria. ${ }^{12}$ In addition to depleting ATP, inhibition of oxidative phosphorylation by Antimycin A has been shown in vivo to produce large quantities of superoxide, a toxic free radical implicated in kidney injury. ${ }^{29}$ Unlike complex I defects, the defects in complex III cause irreversible and severe necrotic injury. This was demonstrated in this study as exposure to Antimycin A for $12 \mathrm{hr}$ caused significant necrotic injury (22\% LDH release above baseline) which increased gradually in a time dependent manner to $61 \%$ at $48 \mathrm{hr}$.

To summarize, Antimycin A with short exposure times produced the most severe injury to renal tubular epithelial cells due to interference with complex III of the electron transport chain, an event that is irreversible and for which there are no compensatory mechanisms. Reduction of oxygen levels to $1 \%$ caused moderate-severe injury but only with extended exposure times indicating basal levels of glycolysis were sufficient to protect cells from necrotic injury. The mild injury produced by 2-Deoxy-D-Glucose was most likely indicative of the ability of RTEC to survive via gluconeogenesis.

This study assessed necrotic cell death. However it is likely that apoptotic cell death occurred at time points where necrosis was not yet evident. Apoptosis is an energy dependent process. Cells undergoing apoptosis are severely damaged, do not function properly, and may eventually undergo necrotic cell death. The contribution of cells initially undergoing apoptosis was not measured in this study and the contribution of apoptotic mechanisms to necrotic cell death as measured by LDH release is unknown. Cell death mechanisms during ischemia are likely a function of time as shown by the results of this study in which the amount of LDH release was determined by the length of exposure to injury-inducing agents rather than the 
concentration of the agent. ATP depletion within a very narrow range determines the mechanism by which cells die. Mouse proximal tubule cells with ATP levels $\leq 15 \%$ of control die uniformly by necrosis whereas those with ATP levels $\geq 25 \%$ of control die of apoptosis. $^{11}$

The methods described in this study provide several advantages. The use of serum-free culture media eliminates cytokines, chemokines and growth factors from the culture media which would be especially advantageous when assessing the efficacy of potential treatment therapies for acute kidney injury, many of which are growth hormones such as epidermal growth factor (EGF), insulinlike growth factor I (IGF-1), hepatocyte growth factor (HGF) and erythropoietin (EPO). The ability to grow renal tubular epithelial cells directly on tissue culture plates without matrix support such as collagen or fibronectin is advantageous as cells can be removed with minimal manipulation, an important consideration if evaluating necrosis or apoptosis by methods such as flow cytometry in which minimizing background cell death is necessary for valid interpretation of results.

Immortalized cell lines such as Madin-Darby canine kidney (MDCK), opossum kidney, porcine cortico-tubular epithelial kidney cells (LLCPK-1) and the human proximal tubule cell line, human kidney 2 (HK-2) have been shown to be more resistant to injury than primary cells. For example, induction of injury using a combination of Antimycin A and 2-Deoxy-D-Glucose produces a 90\% ATP depletion but fails to rapidly kill HK-2 cells. Primary cells may therefore be more representative of in vivo kidney injury. ${ }^{4}$ In this study, the C57B16 strain was used for the culture of RTEC; however the culture method can be applied to genetically manipulated mice and so offers another advantage over the use of cell lines.

The LDH release assay for assessment of necrotic ischemic injury is reproducible, simple to perform and cost effective in terms of equipment and lab disposables. The reaction is carried out in the culture wells and therefore cells do not have to be removed from the plate thus minimizing background cell death. The amount of LDH released into culture supernatants is compared to the total $\mathrm{LDH}$ release per test and therefore inter-well variation in cell number is accounted for and results from individual wells may be directly compared. This makes the interpretation of results objective and easily quantifiable unlike many histologic based methods.

In summary, ischemic injury ranging from mild to moderate to severe was induced in RTEC in vitro by depletion of ATP using Antimycin A and 2-Deoxy-D-Glucose and by reducing the atmospheric oxygen content to $1 \%$ using the hypoxia chamber. Baseline LDH release was consistently low $(\leq 20 \%)$ when cells were cultured in vehicle media and there was no significant difference in baseline LDH release over time $(12-48 \mathrm{hr})$. The ability of all three inducing agents to produce moderate injury indicates the injury was not specific to the metabolic inhibitor used. A simple, reproducible method for induction and assessment of necrotic ischemic injury has therefore been established in which to better understand underlying mechanisms of acute kidney injury and in which to test potential treatment therapies.

\section{Acknowledgements}

We wish to thank Karen Miller and Karen Kinne for critical reading and preparation of this manuscript.

\section{References}

1. Basile DP, Donohoe D, Roethe K, Osborn JL. Renal ischemic injury results in permanent damage to peritubular capillaries and influences long-term function. Am J Physiol Renal Physiol 2001; 281:F887-99.

2. Kaushal GP, Basnakian AG, Shah SV. Apoptotic pathways in ischemic acute renal failure. Kidney Int 2004; 66,:500-6.

3. Lieberthal W, Koh JS, Levine JS. Necrosis and apoptosis in acute renal failure. Semin Nep hrol 1998; 18:505-18.

4. Sheridan AM, Schwartz JH, Kroshian VM, Tercyak AM, Laraia J, Masino S, Lieberthal W. Renal mouse proximal tubular cells are more susceptible than MDCK cells to chemical anoxia. Am J Physiol 1993; 265:F342-50.

5. Molitoris BA, Marrs J. The role of cell adhesion molecules in ischemic acute renal failure. Am J Med 1999; 106:583-92.

6. Bonventre JV, Kelly KJ. Adhesion molecules and acute renal failure. Adv Nep hrol Necker Hosp 1996; 25:159-76.

7. Hallett MA, Dagher PC Atkinson SJ. Rho GTPases show differential sensitivity to nucleotide triphosphate depletion in a model of ischemic cell injury. Am J Physiol Cell Physiol 2003; 285:C129-38.

8. Caron A, Desrosiers RR, Beliveau R. Kidney ischemia-reperfusion regulates expression and distribution of tubulin subunits, beta-actin and rho GTPases in proximal tubules. Arch Biochem Biophys 2004; 431:31-46.

9. Braga VM, Machesky LM, Hall A, Hotchin NA. The small GTPases Rho and Rac are required for the establishment of cadherin-dependent cell-cell contacts. J Cell Biol 1997; 137:1421-31.

10. Lieberthal W, Levine JS. Mechanisms of apoptosis and its potential role in renal tubular epithelial cell injury. Am J Physiol 1996; 271:F477-88.

11. Lieberthal W, Menza SA, Levine JS. Graded ATP depletion can cause necrosis or apoptosis of cultured mouse proximal tubular cells. Am J Physiol 1998; 274:F315-27.

12. Dagher PC. Modeling ischemia in vitro: selective depletion of adenine and guanine nucleotide pools. Am J Physiol Cell Physiol 2000; 279:C1270-7.

13. Weinberg JM. The cell biology of ischemic renal injury. Kidney Int 1991; 39:476-500.

14. Bagnasco S, Good D, Balaban R, Burg M. Lactate production in isolated segments of the rat nep hron. Am J Physiol 1985; 248:F522-6.

15. Sak hrani LM, Badie-Dezfooly B, Trizna W, Mikhail N, Lowe AG, Taub M, et al. Transport and metabolism of glucose by renal proximal tubular cells in primary culture. Am J Physiol 1984; 246:F757-64.

16. Tang MJ, Suresh KR, Tannen RL. Carbohydrate metabolism by primary cultures of rabbit proximal tubules. Am J Physiol 1989; 256:C532-9.

17. Guder WG. Hormonal regulation of renal gluconeogenesis in isolated tubule fragments. Curr Probl Clin Biochem 1976; 6:202-13.

18. Hollenberg MD, Cuatrecasas P. Insulin and epidermal growth factor. Human fibroblast receptors related to deoxyribonucleic acid synthesis and amino acid uptake. J Biol Chem $1975 ; 250: 3845-53$.

19. Dagher PC. Apoptosis in ischemic renal injury: roles of GTP depletion and p53. Kidney Int 2004; 66:506-9.

20. Weinberg JM, Davis JA, Abarzua M, Rajan T. Cytoprotective effects of glycine and glutathione against hypoxic injury to renal tubules. J Clin Invest 1987; 80:1446-54.

21. Weinberg JM, Davis JA, Abarzua M, Smith RK, Kunkel R. Ouabain-induced lethal proximal tubule cell injury is prevented by glycine. Am J Physiol 1990; 258:F346-55.

22. Weinberg JM, Davis JA, Abarzua M, Kiani T, Kunkel R. Protection by glycine of proximal tubules from injury due to inhibitors of mitochondrial ATP production. Am J Physiol 1990; 258:C1127-40.

23. Weinberg JM, Davis JA, Roeser NF, Venkatachalam MA. Role of increased cytosolic free calcium in the pathogenesis of rabbit proximal tubule cell injury and protection by glycine or acidosis. J Clin Invest 1991; 87:581-90.

24. Almeida AR, Wetzels JF, Bunnachak D, Burke TJ, Chaimovitz C, Hammond, et al. Acute phosphate depletion and in vitro rat proximal tubule injury: protection by glycine and acidosis. Kidney Int 1992; 41:1494-500.

25. Weinberg JM, Buchanan DN, Davis JA, Abarzua M. Metabolic aspects of protection by glycine against hypoxic injury to isolated proximal tubules. J Am Soc Nephrol 1991; 1:949-58.

26. Weinberg JM, Davis JA, Abarzua M, Kiani T. Relationship between cell adenosine triphosphate and glutathione content and protection by glycine against hypoxic proximal tubule cell injury. J Lab Clin Med 1989; 113:612-22.

27. Weinberg JM, Davis JA, Roeser NF, Venkatachalam MA. Role of intracellular pH during cytoprotection of proximal tubule cells by glycine or acidosis. J Am Soc Nephrol 1994; 5:1314-23.

28. Weinberg JM, Venkatachalam MA, Roeser NF, Saikumar P, Dong Z, Senter RA, et al. Anaerobic and aerobic pathways for salvage of proximal tubules from hypoxia-induced mitochondrial injury. Am J Physiol Renal Physiol 2000; 279:F927-43.

29. Jones DP. Renal metabolism during normoxia, hypoxia, and ischemic injury. Annu Rev Physiol 1986; 48:33-50. 


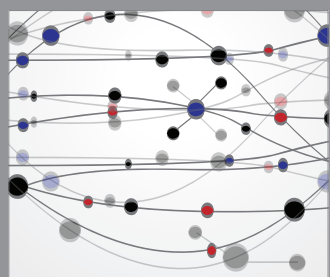

The Scientific World Journal
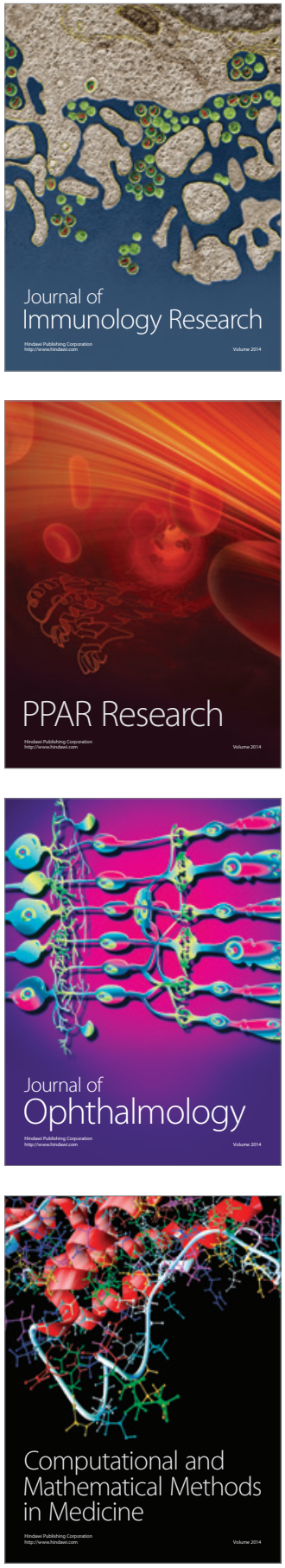

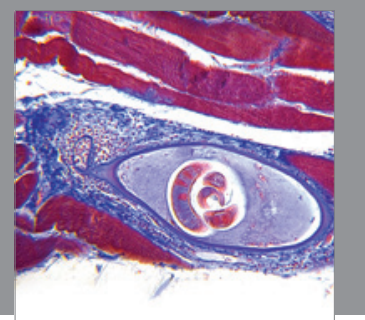

Gastroenterology

Research and Practice
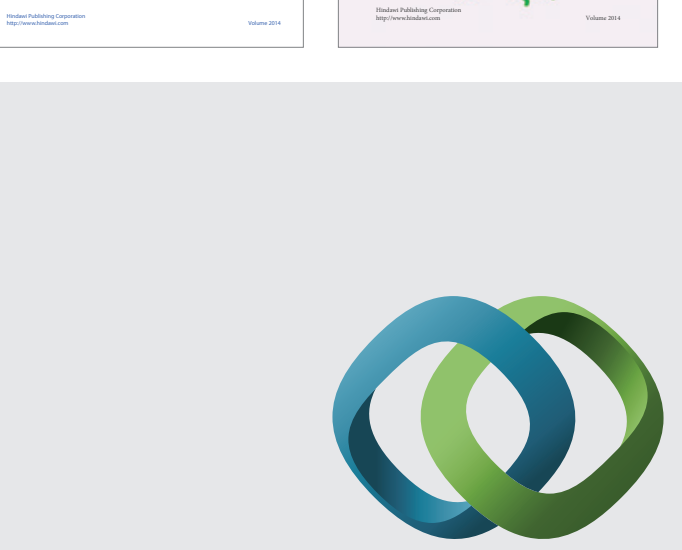

\section{Hindawi}

Submit your manuscripts at

http://www.hindawi.com
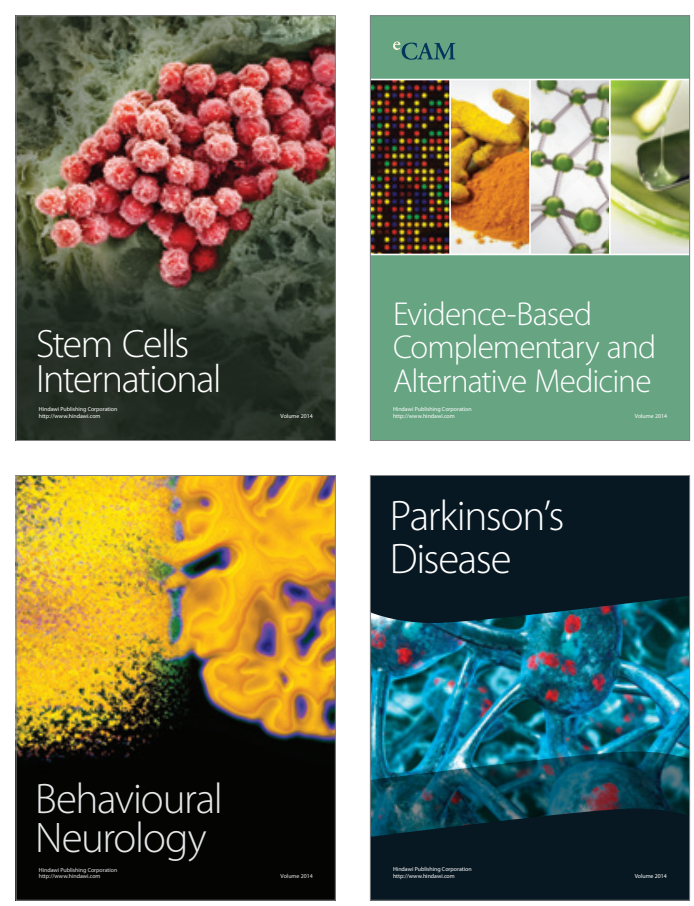

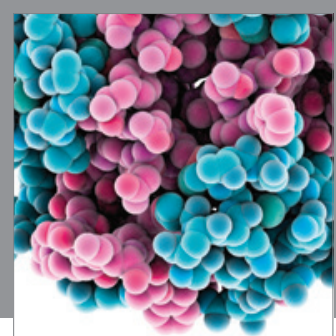

Journal of
Diabetes Research

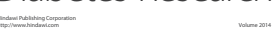

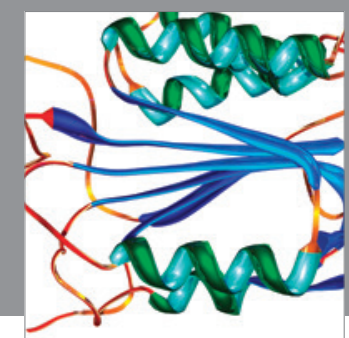

Disease Markers
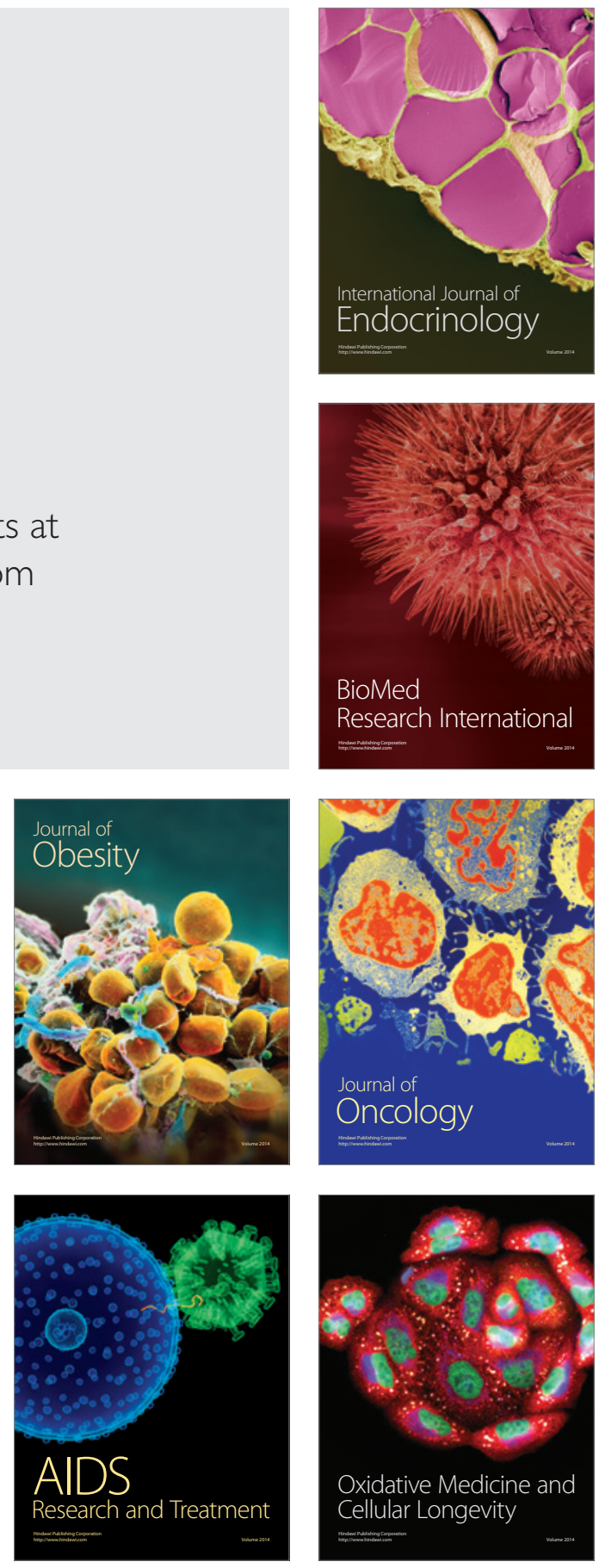